\title{
Zur Relevanz von Polar- und Meerespolitik. Ein Bericht zur Gründung der Themengruppe „Polar- und Meerespolitik“ der Deutschen Vereinigung für Politikwissenschaft an der Universität Bielefeld vom 30. September bis 02. Oktober 2019
}

\author{
Sebastian Knecht • Anja Menzel • Christoph Humrich
}

Online publiziert: 26. Mai 2020

(C) Der/die Autor(en) 2020

\section{Zur Aktualität von Polar- und Meerespolitik}

Polare und marine Räume gewinnen gegenwärtig an sozialer, politischer, wirtschaftlicher und ökologischer Bedeutung. Maßgeblich befördert wird dieser Bedeutungszuwachs durch die Wahrnehmung dieser Räume als neue Schauplätze einer Großmächtekonkurrenz oder als zwischenstaatliche Konfliktzonen in Fragen maritimer Grenzziehung, des Ressourcenzugangs oder der Nutzung sowie Sicherung internationaler Schifffahrtsrouten. Aber auch die zunehmende Übernutzung und Verschmutzung der Polarregionen und Meere sowie ihre dramatischen Veränderungen durch die Erderwärmung schlagen sich im öffentlichen Bewusstsein nieder und stellen Politik und Wissenschaft vor Herausforderungen. In Medien, Politik und wissenschaftlicher Literatur ist daher von den Polar- und Meeresregionen des Öfteren als climate, resource, security oder knowledge frontiers die Rede - schnellem Wandel unterliegende Räume, in denen neue Chancen und Risiken hohen politischen Handlungsdruck für die beteiligten Akteure erzeugen. An der frontier stehen diesem Handlungsdruck aber sowohl ein Mangel an handlungsleitenden Normen, Regeln und Institutionen als auch ein Mangel an gesichertem Wissen als Handlungsgrundlage gegenüber.

Dr. S. Knecht $(\bowtie)$

Fakultät für Soziologie, Universität Bielefeld, Universitätsstraße 25, 33615 Bielefeld, Deutschland E-Mail: sebastian.knecht@uni-bielefeld.de

\section{Dr. A. Menzel}

Fakultät für Kultur- und Sozialwissenschaften, FernUniversität in Hagen, Universitätsstraße 33, 58097 Hagen, Deutschland

E-Mail: anja.menzel@fernuni-hagen.de

Dr. C. Humrich

Fakultät für Geisteswissenschaften, Universität Groningen, Oude Kijk in't Jatstraat 26, 9712

EK Groningen, Niederlande

E-Mail: c.humrich@rug.nl 
Um der Aktualität von Polar- und Meerespolitik von Seiten der Politikwissenschaft Rechnung zu tragen, fand vom 30. September bis 02. Oktober 2019 an der Universität Bielefeld mit finanzieller und logistischer Unterstützung des dortigen Instituts für Weltgesellschaft die Offene Tagung Polar- und Meerespolitik statt. Den Schlusspunkt der Tagung bildete die Konstituierung der Themengruppe „Polar- und Meerespolitik“ der Deutschen Vereinigung für Politikwissenschaft (DVPW). Zuvor wurden insgesamt 16 Papiere zur Polar- und Meerespolitik präsentiert und diskutiert. Den oben skizzierten Implikationen des Begriffs frontier folgend, protokolliert dieser Tagungsbericht die Diskussionen anhand der präsentierten Papiere in drei Abschnitten zu marinen und polaren Akteuren, Ordnungen und Wissen.

\section{Polare und marine Akteure}

Insgesamt sechs Vorträge fokussierten in erster Linie auf die Politiken, mit denen spezifische Akteure den Chancen und Risiken an den jeweiligen frontiers begegnen.

Die Beiträge von Jacqueline Götze (Deutsches Institut für Entwicklungspolitik) und Christoph Humrich (Universität Groningen) einte dabei, dass sie den längerfristigen Wandel von Arktispolitiken zum einen in Bezug zu den Veränderungen der Region setzten, zum anderen aber auch interne Faktoren herausstrichen. Götze zeigte anhand einer Inhaltsanalyse von Dokumenten der Europäischen Union im Zeitraum von 2007 bis 2018 auf, wie die EU ihre Arktisstrategie den sich verändernden Gegebenheiten der Region anpasste, um ihren Einfluss geltend machen zu können. $\mathrm{Zu}$ verstehen seien diese Anpassungen intern aber nur durch eine Kombination von interessen- und normengeleiteter Politik. Humrich arbeitete ebenfalls durch eine Dokumentenanalyse heraus, wie seit der Jahrtausendwende innenpolitische Motive und Dynamiken einen erheblichen Einfluss auf die Arktispolitik Norwegens ausübten.

Russlands Entwicklungsbemühungen in der Arktis thematisierte der Vortrag von Nadir Kinossian (Leibniz-Institut für Länderkunde) am Beispiel des Verwaltungsbezirks Murmansk. Er zeigte unter anderem auf, dass die Regionalpolitik der Russischen Föderation dem wirtschaftlichen Potenzial der Arktis mit zunehmenden Zentralisierungstendenzen begegnet, die mit den lokalen Gegebenheiten und wirtschaftlichen Möglichkeiten der Region kollidieren. Mit Blick auf die externe Dimension russischer Arktispolitik diagnostizierte der Vortrag von Joachim Weber (ChristianAlbrechts-Universität zu Kiel) ein Paradoxon zwischen militärischer Aufrüstung und wirtschaftlichem Infrastrukturaufbau: Die wirtschaftliche Entwicklung im Norden Russlands hängt von regionaler Stabilität und Kooperation in der Arktis ab, die zugleich durch Militarisierung der Region untergraben wird. Der augenscheinliche Widerspruch würde aber vor dem Hintergrund des globalen Großmachtanspruches der Russischen Föderation sowohl als Handlungsdilemma als auch Optimierungsstrategie erkennbar: Der Anspruch lässt sich nur mit ökonomischem und militärischem Wachstum einlösen.

Aus allen vier Präsentationen zu Arktispolitiken lässt sich der Schluss ziehen, dass gängige Darstellungen der Arktis als security and resource frontier die Kontingenzen akteursspezifischer Reaktionen zugunsten vermeintlich systemischer Dynamiken auf der regionalen Ebene vernachlässigen. 
Neben supranationalen und staatlichen Akteuren werden an den frontiers der Polar- und Meeresregionen aber auch nichtstaatliche Akteure in dem Maße aktiv, wie Risiken eine breitere Öffentlichkeit alarmieren oder Chancen auf private Gewinne Unternehmen interessieren. Aletta Mondré (Christian-Albrechts-Universität zu Kiel) beleuchtete in dieser Hinsicht die verstärkte Einbindung und Aktivitäten von Nichtregierungsorganisationen (NROs) in der Internationalen Meeresbodenbehörde. Zwar seien NROs zunehmend repräsentiert, ihr Einfluss auf internationale Verhandlungen im Bereich Tiefseebergbau sei aber nach wie vor formal stark eingeschränkt. Ulrike Kronfeld-Goharani (Christian-Albrechts-Universität zu Kiel) befasste sich in ihrer diskursanalytischen Untersuchung mit Nachhaltigkeitsstrategien in der maritimen Wirtschaft. Sie untersuchte zu diesem Zweck insgesamt 396 Unternehmen auf deren Verpflichtung auf Umwelt- und Sozialstandards. Im Ergebnis konstatierte sie eine zunehmende Verbindlichkeit von Nachhaltigkeitsstandards. Diese hätten jedoch vor allem das Ziel, durch Nichthandeln langfristig entstehende Kosten zu reduzieren.

\section{Polare und marine Ordnungen}

Der Frontier-Charakter der Polar- und Meeresregionen bedingt ein System internationaler Governance, in dem einzelne Politikbereiche ebenso wie geografische Teilgebiete bisher nicht erfasst sind. Institutionalisierte ebenso wie informelle Formen der Governance sind aktuell jedoch im Entstehen begriffen, während sich bestehende Arrangements im Prozess der Anpassung an die dramatischen Veränderungen der Räume kontinuierlich wandeln. Diese Prozesse verlaufen weder einheitlich noch geradlinig. Die emergente, nichthierarchische Ordnung ist durch ein hohes Maß institutioneller Fragmentierung gekennzeichnet und wird begleitet von politischen Kontroversen um ihre Repräsentativität, Legitimität, Autorität und Effektivität.

In diesem Kontext sah der Vortrag von Dorothea Wehrmann (Deutsches Institut für Entwicklungspolitik) den Arktischen Rat trotz einiger prozeduraler Schwächen aufgrund seiner Inklusivität, vertrauensfördernden Vermittlerrolle und institutionellen Flexibilität als Vorbild für transnationale Kooperation an. Dieses Vorbild könne auch in Überlegungen zur Bereitstellung globaler Gemeingüter, insbesondere in der Entwicklungszusammenarbeit im Rahmen der Agenda 2030 und der internationalen Klimapolitik, herangezogen werden.

Um die unter Anpassungsdruck stehenden Ordnungen europäischer Fischereigovernance ging es in den Vorträgen von Hubert Zimmermann (Universität Marburg) und Johanna Ferretti (Thünen-Institut, Rostock). Zimmermann untersuchte den Erfolg der EU, die Normen der Gemeinsamen Fischereipolitik zu externalisieren. Dazu zeichnete er die Diffusionsmechanismen nach, durch welche die EU Einfluss auf die Standards internationaler Fischereipolitik nimmt. Die EU ist dabei durchaus erfolgreich, Ansprüche von good governance und Nachhaltigkeit als Ordnungsprinzipien auch global zu verankern - wobei Sanktionsmöglichkeiten eine entscheidende Rolle spielen. Im Fokus von Ferrettis Vortrag stand hingegen die interne Ordnung der EU. Ferretti berichtete von einem Stakeholder-Workshop zu Regionalisierungsoptionen, der im Juli 2018 in Brüssel stattfand. In der Bewertung der Optionen verdeutlichte sie, dass eine weitere Regionalisierung der EU-Fischereipolitik die Flexibilität in 
den Regionen erhöhen und die Entscheidungsfindung vereinfachen würde. Für eine erfolgreiche Fischereigovernance müsse zum moving down jedoch auch ein gleichzeitiges moving up erfolgen, also eine verstärkte Präsenz von Fischereithemen in höheren EU-Gremien.

Um globale Schifffahrtsordnungen ging es in den Vorträgen von Christian Wirth (German Institute of Global and Area Studies) und Anja Menzel (FernUniversität in Hagen). Wirth analysierte in seinem Vortrag die konfliktive Bildung maritimer Ordnung im Indopazifik. Er wies darauf hin, dass sich das historische Spannungsverhältnis zwischen zwei zentralen Prinzipien oder Normen des Seerechtsübereinkommens (SRÜ) der Vereinten Nationen, der Freiheit der Schifffahrt und der territorialen Souveränität, in der letzten Dekade stark intensiviert hat. Die Ausbeutung mariner Ressourcen und der Schutz der freien Schifffahrt unter dem Primat der nationalen Sicherheit führten zur Politisierung des Seerechts und beschränkten, paradoxerweise, die Freiheit der Schifffahrt. Menzel untersucht hingegen kooperative Ordnungsbildung zur Bekämpfung maritimer Kriminalität. Sie argumentierte, dass die fragmentierte institutionelle Landschaft Staaten zwar forum shopping ermögliche, dies aber nicht notwendigerweise negative Konsequenzen habe. Vielmehr zeige das Beispiel der Pirateriebekämpfung in Asien, dass sich eine Arbeitsteilung zwischen zentralen Institutionen entwickele, deren Kompetenzen und Aufgabenbereiche sich ergänzen.

\section{Polares und marines Wissen}

An den frontiers mariner und polarer Räume fehlt gesichertes Wissen als Handlungsgrundlage. Diese Wissenslücken zu beheben ist ebenso aufwändig wie kostspielig. Einerseits begünstigt das unkooperative Strategien der unmittelbaren Kostenvermeidung oder Gewinnrealisierung, für die auch die Wissenschaft eingespannt wird. Andererseits kommt es angesichts der grenzüberschreitenden Wirkung vieler Problemstellungen internationaler Polar- und Meeresgovernance in der Wissensproduktion zu enger Kooperation an der knowledge frontier unter Beteiligung staatlicher wie nichtstaatlicher Akteure.

Dass Politik und Wissenschaft sich daher gegenseitig durchdringen, wurde im Vortrag von Martin Weiss (Deutsches Schifffahrtsmuseum) an einem historischen Beispiel deutlich. Er zeigte an der ersten deutschen hydrographischen Expedition im Atlantik auf, wie der politische Kontext nach dem Ersten Weltkrieg die deutsche Wissenschaft und Marine zusammenbrachte und die Entwicklung eines hydrographischen Forschungsprogramms begünstigte. Die Durchführung dieses Programms wurde auch insofern zu einem politischen Ereignis, als sie vom Kapitän des Forschungsschiffes für nationalistische Kundgebungen mit Auslandsdeutschen benutzt wurde.

Dem Problem der Bereitstellung von Wissen in Kontexten mariner und polarer Governance wandten sich die Beiträge von Ina Tessnow von Wysocki (Universität Wien) und Annegret Kuhn (Christian-Albrechts-Universität zu Kiel) zu. Tessnow von Wysocki fragte in einem mit Alice Vadrot (Universität Wien) verfassten Literaturüberblick danach, wie in Fachveröffentlichungen die Rolle der Wissenschaft für die Governance mariner Biodiversität jenseits der Grenzen nationaler Jurisdiktion 
deskriptiv erfasst oder präskriptiv definiert wird. Dabei wurde die herausragende Bedeutung wissenschaftlicher Erkenntnisse für die entsprechenden Institutionen, ihre Politiken und politischen Prozesse betont. Ebenso ergab sich, dass von wissenschaftlicher Seite erkannt wird, wie strukturelle Ungleichgewichte von Datenverfügbarkeit, Forschungskapazitäten und -ausrichtung Wissensproduktion politisch aufladen. Der Beitrag von Kuhn verhielt sich dazu insofern komplementär, als er auf die Probleme einging, die sich bei der Integration indigenen bzw. traditionellen Wissens in Strukturen mariner Governance und ihrer Wissensproduktion ergeben.

Reflexiv bezogen auf die entsprechende Wissensproduktion der bei der Tagung vorwiegend vertretenen politikwissenschaftlichen Teildisziplin der Internationalen Beziehungen (IB) waren schließlich zwei Papiere. Sebastian Knecht (Universität Bielefeld) untersuchte mit einer bibliometrischen Analyse die epistemologische, theoretische und methodische Ausrichtung von Veröffentlichungen zur Arktispolitik in wissenschaftlichen Fachzeitschriften. Dabei zeigte sich, dass deskriptive Analysen und Einzelfallstudien gegenüber theoriegeleiteter und vergleichender Forschung überwiegen, was auch einen Erklärungsansatz dafür liefert, warum die Arktis eine Randposition in der IB-Forschung einnimmt und nicht als relevanter Fall für generalisierbare Erkenntnisse wahrgenommen wird. Hannes Hansen-Magnusson (Universität Cardiff) stellte demgegenüber heraus, dass aus der Arktispolitik gerade darum interessante generalisierende Erkenntnisse für die Disziplin zu gewinnen seien, weil hier die dramatischen Entwicklungen im Zeitalter des Anthropozäns besonders zutage treten. Mit Blick auf drei verschiedene Aspekte regionaler Kooperation in der Arktis zeigte Hansen-Magnusson dementsprechend, dass sich an der arktischen climate frontier die global relevanten und in den traditionellen IB-Theorien vernachlässigten Verstrickungen zwischen sozialer Welt und Natur in ihren Konsequenzen für die Prozesse internationaler Politik offenbaren.

\section{Zur Relevanz politikwissenschaftlicher Polar- und Meeresforschung}

Auf der offenen Tagung zur Polar- und Meerespolitik in Bielefeld zeigte sich, dass an den frontiers der Polar- und Meeresregionen mindestens drei interessante Widersprüche im Zentrum politischer Prozesse und im Mittelpunkt politikwissenschaftlicher Auseinandersetzung mit Fragen polarer und mariner Politik stehen. Beflügelt durch die Bestimmungen des 1994 in Kraft getretenen SRÜ der UN lässt sich im Hinblick auf marine Ordnungen erstens eine Verschiebung der Grenzen staatlicher Autoritätsausweitung in Meeresgebiete beobachten, die bis dato jenseits der Grenzen nationaler Jurisdiktion lagen. Dem widersprechen nicht nur die faktischen (technischen) Grenzen der Autoritätsausübung in Meeren und Polargebieten als Räumen begrenzter Staatlichkeit (in Anlehnung an den gleichnamigen Sonderforschungsbereich 700), sondern auch ihre prominente normative Kontextualisierung als internationale Gemeinschaftsräume und gemeinsames Erbe der Menschheit. An diesen Widerspruch von Territorialisierung und Transnationalität knüpft sich ein zweiter in der Akteursdimension an - das Spannungsverhältnis von marinen und polaren Räumen als Projektionsfläche globaler Machtansprüche und der gleichzeitigen Anerkennung, dass Gemeingüter wie marine und polare Umwelt, natürliche Ressourcenbestände, 
die Sicherheit der Seefahrt und wissenschaftliche Erforschung einem besonderen Schutzbedürfnis unterliegen und folglich Kooperation auch zur Erreichung je eigener Ziele unausweichlich ist. Im Hinblick auf die Wissensproduktion an der marinen und polaren knowledge frontier haben die Vorträge der Tagung drittens gezeigt, dass dem Bedarf gesicherten Wissens die politische Verstrickung der Wissenschaft in die Quere kommen kann, also Forschungsergebnisse teilweise unter Bedingungen produziert werden, die dazu Anlass geben, an der Objektivität und daher Legitimität mit ihnen einhergehender politischer Forderungen zu zweifeln.

Um anzuerkennen, dass einerseits die Entwicklungen in den Polar- und Meeresregionen von großer politischer Aktualität und Relevanz sind, und sich aus den mit ihnen befassten Politiken andererseits ein auch über diese Räume hinausgehendes Interesse der Politikwissenschaft begründen lässt, muss man nicht den hier verwendeten Begriff der frontier teilen. Geeignete Begriffe und Konzepte zu finden, um sich Themen der Polar- und Meerespolitik systematisch zuzuwenden und sie für das breitere Fach fruchtbar zu machen sowie sie auch für die praktische Politik darzustellen, verfolgt die neu gegründete Themengruppe „Polar- und Meerespolitik“ der DVPW als ihr Ziel. Vor diesem Hintergrund soll die Themengruppe nach innen eine Koordinierungsfunktion für an politikwissenschaftlichen Fragestellungen mit Polar- und Meeresbezug Interessierten übernehmen und nach außen die Sichtbarkeit des Feldes und der vorhandenen wissenschaftlichen Expertise erhöhen. Als Sprecher*innen der Themengruppe wurden einstimmig Prof. Mathias Albert (Universität Bielefeld), Dr. Christoph Humrich (Universität Groningen), Dr. Anja Menzel (FernUniversität in Hagen) und Prof. Aletta Mondré (Christian-Albrechts-Universität zu Kiel) gewählt. Eine Website und Mailingliste ist über die Homepage der DVPW zu erreichen.

Funding Open Access funding provided by Projekt DEAL.

Open Access Dieser Artikel wird unter der Creative Commons Namensnennung 4.0 International Lizenz veröffentlicht, welche die Nutzung, Vervielfältigung, Bearbeitung, Verbreitung und Wiedergabe in jeglichem Medium und Format erlaubt, sofern Sie den/die ursprünglichen Autor(en) und die Quelle ordnungsgemäß nennen, einen Link zur Creative Commons Lizenz beifügen und angeben, ob Änderungen vorgenommen wurden.

Die in diesem Artikel enthaltenen Bilder und sonstiges Drittmaterial unterliegen ebenfalls der genannten Creative Commons Lizenz, sofern sich aus der Abbildungslegende nichts anderes ergibt. Sofern das betreffende Material nicht unter der genannten Creative Commons Lizenz steht und die betreffende Handlung nicht nach gesetzlichen Vorschriften erlaubt ist, ist für die oben aufgeführten Weiterverwendungen des Materials die Einwilligung des jeweiligen Rechteinhabers einzuholen.

Weitere Details zur Lizenz entnehmen Sie bitte der Lizenzinformation auf http://creativecommons.org/ licenses/by/4.0/deed.de. 